Publications of the Astronomical Society of the Pacific, 110:1336-1341, 1998 November

(C) 1998. The Astronomical Society of the Pacific. All rights reserved. Printed in U.S.A.

\title{
An 11.6 Micron Keck Search for Exo-Zodiacal Dust
}

\author{
Marc J. Kuchner, Michael E. Brown, and Chris D. Koresko \\ California Institute of Technology, Pasadena, CA 91125; mjk@gps.caltech.edu, mbrown@gps.caltech.edu, koresko@gps.caltech.edu \\ Received 1998 August 7; accepted 1998 August 12
}

\begin{abstract}
We have begun an observational program to search nearby stars for dust disks that are analogous to the disk of zodiacal dust that fills the interior of our solar system. We imaged six nearby main-sequence stars with the Keck telescope at $11.6 \mu \mathrm{m}$, correcting for atmosphere-induced wavefront aberrations and deconvolving the point spread function via classical speckle analysis. We compare our data to a simple model of the solar zodiacal dust based on COBE/DIRBE observations and place upper limits on the concentration of exo-zodiacal dust around these stars.
\end{abstract}

\section{INTRODUCTION}

Our Sun is surrounded by a disk of warm $(>150 \mathrm{~K})$ "zodiacal" dust that radiates most of its thermal energy at 10-30 $\mu \mathrm{m}$. This dust is produced largely in the inner part of the solar system by collisions in the asteroid belt (Dermott et al. 1992) and cometary outgassing (Liou \& Zook 1996). Zodiacal dust is interesting as a general feature of planetary systems, and as an indicator of the presence of larger bodies that supply it; dust orbiting a few AU from a star is quickly removed as it loses angular momentum to Poynting-Robertson drag (Robertson 1937). Understanding the extrasolar analogs of zodiacal dust may also be crucial in the search for extrasolar planets (Beichman 1996) since exo-zodiacal dust in a planetary system could easily outshine the planets and make them much harder to detect.

The best current upper limits for the existence of exo-zodiacal dust disks come from IRAS measurements of 12 and 25 micron excesses above photospheric emission. Seen from a nearby star, solar system zodiacal dust would create only a $10^{-4}$ excess over the Sun's photospheric emission at $20 \mu \mathrm{m}$. IRAS measurements, however, have typical measurement errors of 5 percent (Moshir et al. 1992) and display systematic offsets of a similar magnitude when they are compared to other photometry (Cohen et al. 1996). If there were a solar-type zodiacal disk with 1000 times the density of the disk around the Sun around Tau Ceti, the nearest $\mathrm{G}$ star, the excess infrared emission would barely exceed the formal $68 \%$ confidence intervals of the IRAS photometry. Moreover, all photometric detection schemes of this sort are limited by how accurately the star's mid-infrared photospheric emission is known. For farther, fainter stars than Tau Ceti, inferring the presence of dust from the IRAS data becomes still harder.

The detection of faint exo-zodiacal dust emission is more feasible if one can resolve the dust emitting region. The high resolution and dynamic range needed for these observations will generally require large interferometers like the Keck Interferometer, the Large Binocular Telescope, and the Very Large Telescope Interferometer. But it is already possible to resolve the zodiacal dust mid-infrared emitting regions of the nearest stars. A $10 \mathrm{~m}$ telescope operating at $12 \mu \mathrm{m}$ has a diffraction-limited resolution of 0.25 , corresponding, for example, to a transverse distance of $2 \mathrm{AU}$ at $8 \mathrm{pc}$.

We have begun a search for zodiacal dust around the nearest stars using the mid-infrared imaging capabilities of the Long Wavelength Spectrometer (LWS) (Jones \& Peutter 1993) on the W. M. Keck telescope. The large aperture of the telescope allows us to make spatially resolved images of the zodiacal dust 11.6 micron emitting region around the stars so that we can look for dust emission above the wings of the point-spread function (PSF) rather than as a tiny photometric excess against the photosphere. We present here the results of two nights of observations and compare them with a simple model of exozodiacal thermal emission to place upper limits on the amount of dust present in the systems we observed.

\section{OBSERVATIONS}

We observed six nearby stars with LWS on the W. M. Keck telescope on 1996 August 3 and 4 using standard mid-infrared imaging techniques. The target stars were the nearest $\mathrm{A}-\mathrm{K}$ main-sequence stars observable from Mauna Kea on those dates. With the object on-axis, we took a series of frames lasting $0.8 \mathrm{~ms}$ each, chopping the secondary mirror between the object and blank sky $8^{\prime \prime}$ to the north at a frequency of $10 \mathrm{~Hz}$. Then we nodded the primary mirror for the next series of frames so that the sky was on-axis and the object off-axis. We repeated this process for three nods over a period of 5 minutes, for an on-source integration time of 1.1 minutes, and a typical noise of $2 \mathrm{mJy}$ in one $0^{\prime \prime} 11$ by $0^{\prime \prime} .11$ pixel due to the thermal background. The seeing was poor both nights, up to $2^{\prime \prime}$ in the visible. To measure the atmosphere-telescope transfer function, we 


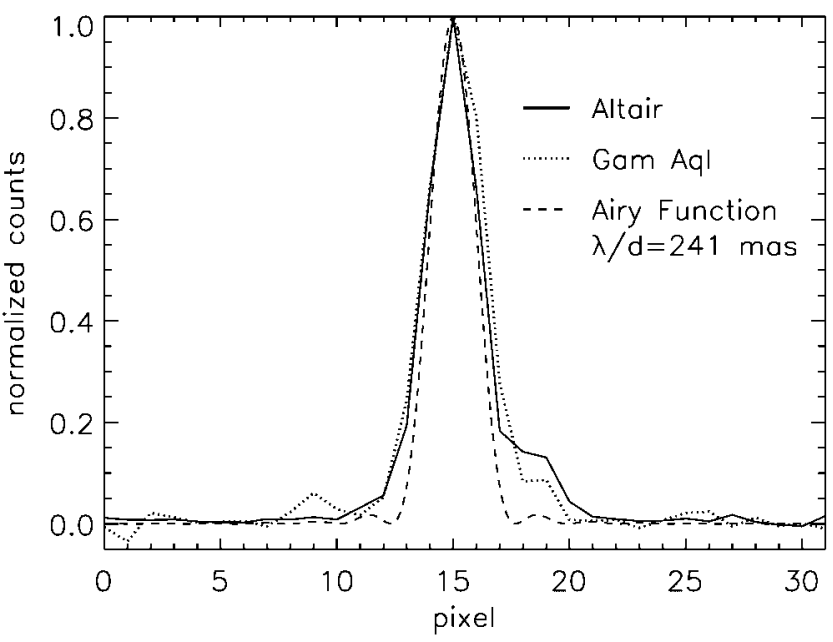

FIG. 1.-Cut through a single $4 \mathrm{~ms}$ image of Altair, compared to a similar cut through an image of a calibrator star, Gamma Aquila, and an Airy function representing the PSF of an ideal, filled, $10 \mathrm{~m}$ aperture at $11.6 \mu \mathrm{m}$. The cores of the images are diffraction-limited, but the wings are sensitive to the instantaneous seeing, making speckle analysis necessary.

made similar observations of seven distant, luminous calibrator stars near our targets on the sky, alternating between target and calibrator every 5-10 minutes.

We increased our frame-rate for the second night of observations so that we could compensate for the seeing using speckle analysis. Figure 1 shows a cut through a single $84 \mathrm{~ms}$ exposure of Altair on August 4, compared to an Airy function representing the diffraction-limited PSF of a filled $10 \mathrm{~m}$ aperture at $11.6 \mu \mathrm{m}$. The cores of the images are diffractionlimited, but the wings are sensitive to the instantaneous seeing, making speckle analysis necessary. Table 1 provides a summary of our observations.

We flat-fielded the images by comparing the response of each pixel to the response of a reference pixel near the center of the detector. First we plotted the data number (DN) recorded by a given pixel against the DN in the reference pixel for all the frames in each run. Since the response of each pixel is approximately linear over the dynamic range of our observations and most of the signal is sky background, which varies with time but is uniform across the chip, the plotted points for each pixel describe a straight line; if all the pixels had the same response, the slope of each line would equal 1 . We divided each pixel's DN by the actual slope of its response curve relative to the reference pixel, effectively matching all pixels to the reference pixel. We then interpolated over bad pixels, frame by frame.

To compensate for the differences in the thermal background between the two nod positions, we averaged together all the on-axis sky frames to measure the on-axis thermal background and subtracted this average from all of the on-axis framesboth object and sky. We used the same procedure to correct the off-axis frames.

Next, we chose subframes of 32 by 32 pixels on each image, centered on the star (or for sky frames, the location of the star in an adjacent object frame), and processed these according to classical speckle analysis (Labeyrie 1970). We Fourier transformed them, and summed the power spectra, yielding a sky power spectrum and an object power spectrum for each series. Then we azimuthally averaged the power spectra in the $u-v$ plane-that is, we averaged over all the frequency vectors of a given magnitude, $\left(u^{2}+v^{2}\right)^{1 / 2}$. This azimuthal averaging corrects for the rotation of the focal plane of the alt-az-mounted Keck telescope with respect to the sky. We then subtracted from every object power spectrum the corresponding sky power spectrum and divided each corrected target power spectrum by the corrected power spectrum of a calibrator star observed in the same manner as the target star immediately before or after the target star. Figure 2 shows an azimuthally averaged power spectrum of Altair and the corresponding sky power spectrum, compared with a power spectrum of calibrator Gamma Aquila and its corresponding sky power.

We then averaged all the calibrated power spectra for a given target. If the object and calibrator are both unresolved, the average calibrated power spectrum should be the power spec-

TABLE 1

OBSERVATIONS

\begin{tabular}{|c|c|c|c|c|c|c|}
\hline Date & Target & Calibrator & $\begin{array}{l}\text { Exposure Time } \\
\text { Per Frame (ms) }\end{array}$ & $\begin{array}{l}\text { Object } \\
\text { Frames }\end{array}$ & Pairs & $\begin{array}{l}\text { Log Disk Density } \\
\text { Solar Disk }=0^{\mathrm{a}}\end{array}$ \\
\hline \multirow[t]{6}{*}{ August 3} & \multirow[t]{2}{*}{ Vega } & R Lyr & 800 & 90 & 2 & \multirow[t]{2}{*}{$\leq 4.0$} \\
\hline & & $\kappa \mathrm{Lyr}$ & 800 & 90 & 1 & \\
\hline & 61 Cyg A & $\zeta$ Cyg & 800 & 90 & 4 & $\ldots$ \\
\hline & 61 Cyg B & $\zeta$ Cyg & 800 & 90 & 5 & $\ldots$ \\
\hline & \multirow[t]{2}{*}{$\tau$ Cet } & $\nu$ Cet & 800 & 90 & 2 & \multirow[t]{2}{*}{$\cdots$} \\
\hline & & & 210 & 348 & 1 & \\
\hline \multirow[t]{4}{*}{ August 4} & \multirow[t]{2}{*}{70 Oph B } & $\beta \mathrm{Oph}$ & 84 & 864 & 5 & \multirow[t]{2}{*}{$\ldots$} \\
\hline & & 74 Oph & 84 & 864 & 4 & \\
\hline & \multirow[t]{2}{*}{ Altair } & $\gamma \mathrm{Aql}$ & 84 & 864 & 6 & \multirow[t]{2}{*}{$\leq 3.2$} \\
\hline & & $\beta$ Aql & 84 & 864 & 5 & \\
\hline
\end{tabular}

${ }^{\text {a }}$ Based on the $C O B E / D I R B E$ model of the solar zodiacal cloud (Kelsall et al. 1998). 


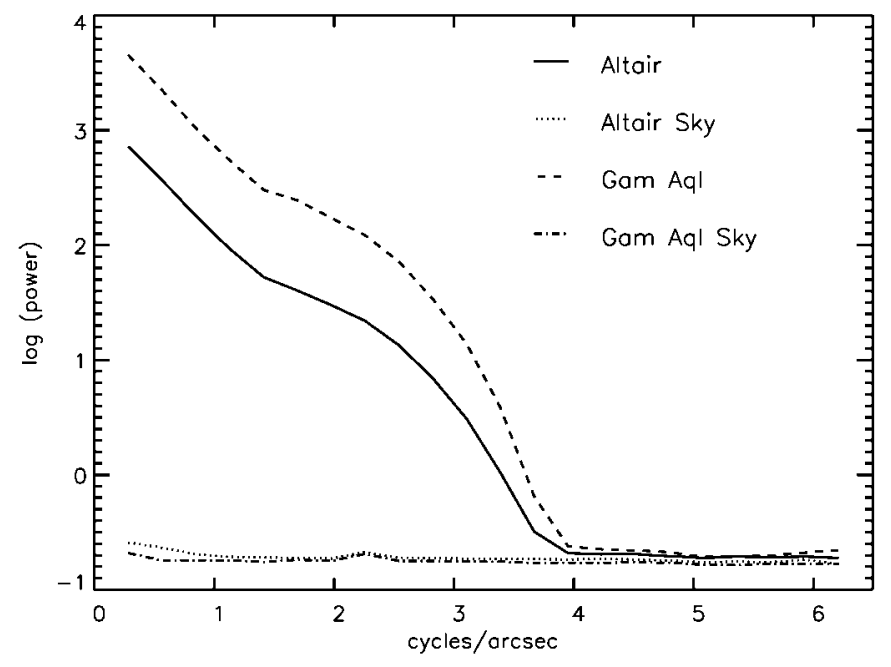

FIG. 2.-Azimuthally averaged power spectrum of Altair and the corresponding sky power spectrum, compared with a power spectrum of calibrator Gamma Aquila and its corresponding sky power. The power in the star images approaches the sky power near the diffraction limit at 4 cycles $\operatorname{arcsec}^{-1}$.

trum of the delta function: a constant. We found that the pixels along the $u$ and $v$ axes of the power spectra were often contaminated by noise artifacts from the detector amplifiers, so we masked them out.

Figures 3 and 4 show the calibrated azimuthally averaged power spectra for our target stars. To compare different power spectra from the same target, we normalized each azimuthally averaged power spectrum so that the geometric mean of the first 10 data points in each spectrum equals 1 .

For Altair and 61 Cygni A and B we had more than three pairs of target and calibrator observations, i.e., calibrated power spectra, so we show the average of all the spectra and error bars representing the $68 \%$ confidence interval for each datum, estimated from the variation among the individual power spectra. The error is primarily due to differences in the atmospheretelescope transfer function between object and calibrator. None of the calibrated power spectra deviate from a straight line by more than a typical error; all the targets are unresolved to the accuracy of a our measurements.

\section{DISCUSSION}

To interpret our observations we compared them to models of the IR emission from the solar zodiacal cloud. We constructed a model for exo-zodiacal emission based on the smooth component of the Kelsall et al. (1998) model of the solar system zodiacal cloud as seen by $C O B E / \mathrm{DIRBE}$, with emissivity $\epsilon \propto r^{-0.34}$ and a temperature $T=286 \mathrm{~K} r^{-0.467} L^{0.234}$, where $r$ is the distance from the star in $\mathrm{AU}$, and $L$ is the luminosity of the star in terms of $L_{\odot}$. For a dust cloud consisting entirely of a single kind of dust particle of a given size and albedo, the $L$ exponent in the expression for the temperature is simply $-\frac{1}{2}$ times the $r$ exponent (Backman \& Paresce 1993).

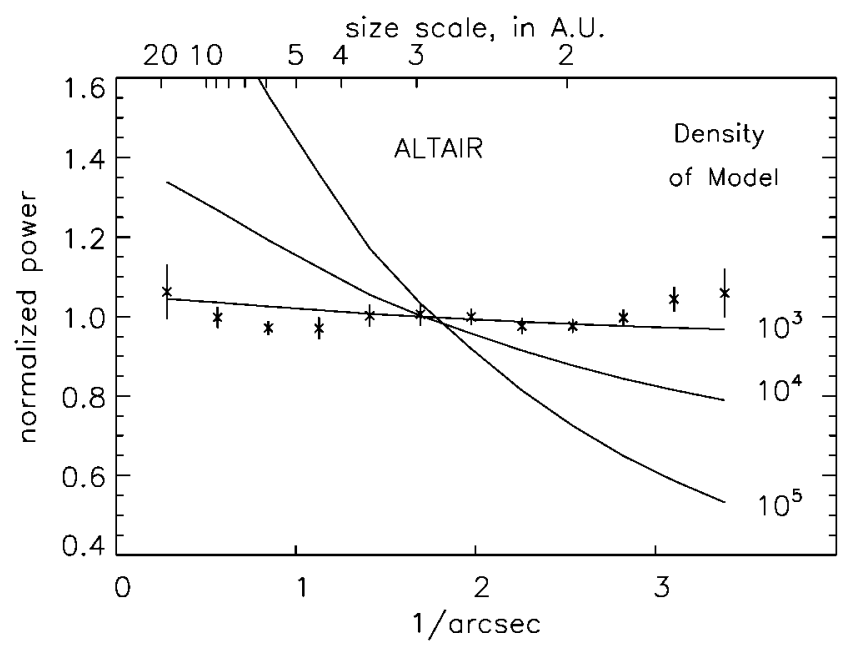

FIG. 3.-Azimuthally integrated power spectrum of Altair compared to simulated power spectra of model disks with various densities $(1=$ the solar disk). An unresolved point source would appear as a straight line at a normalized power of 1.0. The densest model disk consistent with the observations has a density of roughly $10^{3}$ times that of the solar disk.

The physics of the innermost part of the solar zodiacal dust is complicated (see Mann \& MacQueen 1993), but our results are not sensitive to the details, because the hottest dust is too close to the star for us to resolve. We assume that the dust sublimates at a temperature of $1500 \mathrm{~K}$, and allow this assumption to define the inner radius of the disk. We set the outer radius of the model to $3 \mathrm{AU}$, the heliocentric distance of the inner edge of our own main asteroid belt. Our conclusions are not sensitive to this assumption; decreasing the outer radius to $2 \mathrm{AU}$ or increasing it to infinity makes a negligible difference in the visibility of the model, even for A stars.

The assumed surface density profile, however, does make a difference. A collisionless cloud of dust in approximately circular orbits spiraling into a star due to Poynting-Robertson drag that is steadily replenished at its outer edge attains an equilibrium surface density that is independent of radius (Wyatt \& Whipple 1950; Briggs 1962). Models that fit data from the Helios space probes (Leinert et al. 1981), the fit by Kelsall et al. (1998) to the DIRBE measurements, and J. Good's (1997, private communication) revised fit to the IRAS data all have surface densities that go roughly as $r^{-0.4}$. This distribution appears to continue all the way in to the solar corona (MacQueen $\&$ Greeley 1995). We find that in general, if we assume an $r^{-\alpha}$ surface density profile, our upper limit for the 1 AU density of a given disk scales roughly as $10^{\alpha / 2}$; disks with more dust toward the outer edge of the 11.6 micron emitting region are easier to resolve.

Likewise, the assumed temperature profile strongly affects our upper limits. Unfortunately, we know little about the temperature profile of the solar zodiacal cloud. DIRBE and IRAS only probed the dust thermal emission near $1 \mathrm{AU}$, and Helios 

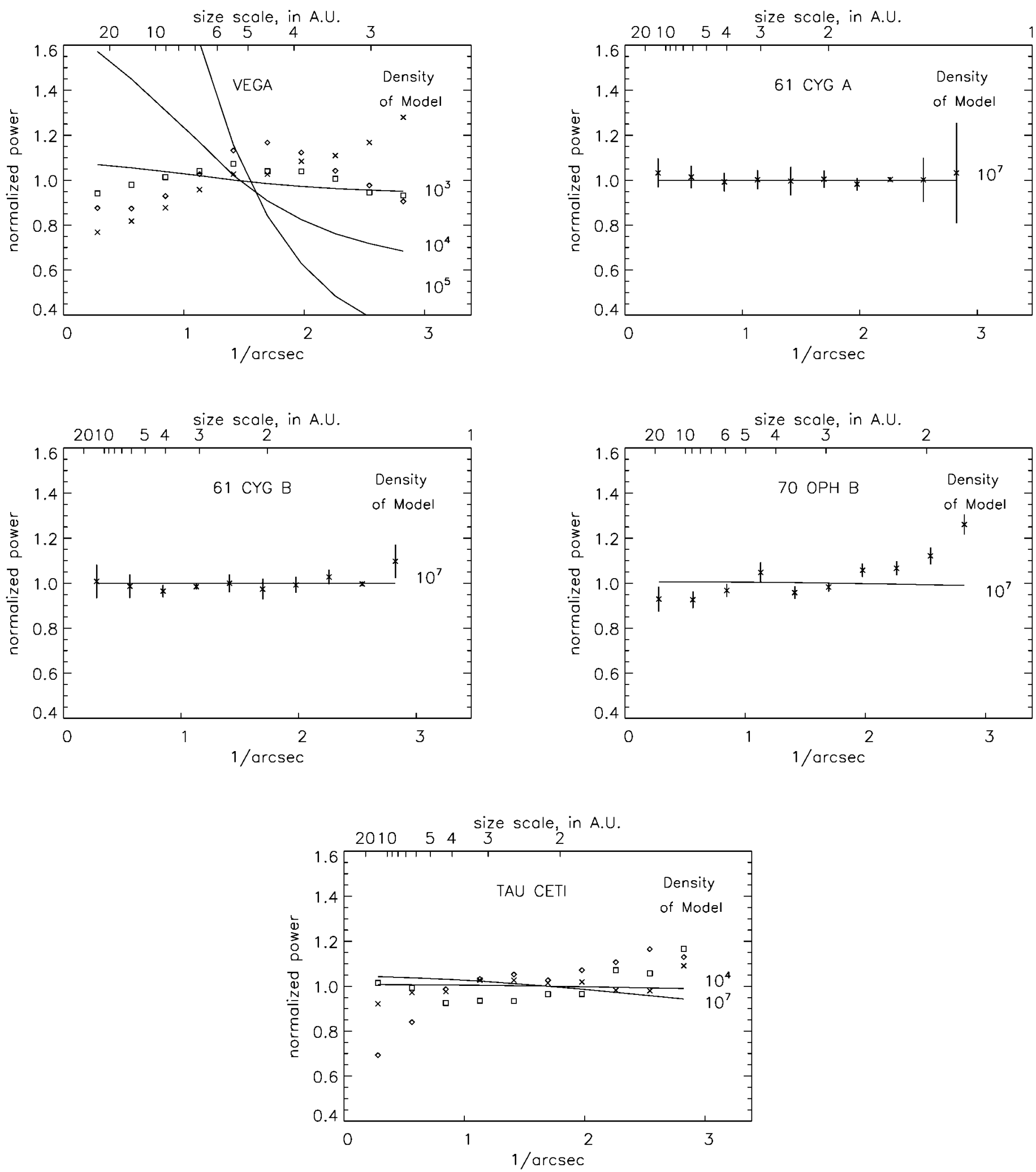

FIG. 4.-Azimuthally integrated power spectra of other target stars compared to simulated power spectra of model disks with various densities 
measured the solar system cloud in scattered light, which does not indicate the dust temperature. We found that a dust cloud model with the IRAS temperature profile $(T=266 \mathrm{~K}$ $r^{-0.359} L^{0.180}$ ) was much easier to resolve than the model based on DIRBE measurements that we present here, especially for $\mathrm{G}$ and $\mathrm{K}$ stars.

To compare the models with the observations, we synthesized high resolution images of the model disks at an inclination of $30^{\circ}$. We calculated the IR flux of the stars from the blackbody function, and obtained the parallaxes of the stars from the Hipparcos Catalog (ESA 1997). We inferred stellar radii and effective temperatures for each star from the literature and checked them by comparing the blackbody fluxes to spectral energy distributions based on photometry from the SIMBAD database (Egret, Wenger, \& Dubois 1991). For Altair and Vega, we use the interferometrically measured angular diameters (Hanbury Brown, Davis, \& Allen 1974) (they are $2.98 \pm$ 0.14 mas and 3.24 mas). Stellar fluxes typically disagree with fitted blackbody curves by $\sim 10 \%$ in the mid-infrared (Engelke 1990), but our method does not require precise photometry, and the blackbody numbers suffice for determining conservative upper limits. We computed the power spectra of the images and normalized them just like the observed power spectra. In Figures 3 and 4, the azimuthally averaged power spectra for our target stars are compared to the extrapolated DIRBE model at a range of model surface densities. Disks with masses as high as $10^{3}$ times the mass of the solar disk will suffer collisional depletion in their inner regions, so they are unlikely to have the same structure as the solar disk. By neglecting this effect we are being conservative in our mass limits. The density of the densest model disk consistent with the data in each case is listed in Table 1.

Altair.-Our best upper limit is for Altair (spectral type A7, distance $5.1 \mathrm{pc}$ ); with 11 pairs of object and calibrator observations we were able to rule out a solar-type disk a few times $10^{3}$ as dense as our zodiacal cloud. Such a disk would have been marginally detectable by IRAS as a photometric excess.

Vega.-IRAS detected no infrared excess in Vega's spectral energy distribution at $12 \mu \mathrm{m}$, with an uncertainty of $0.8 \mathrm{Jy}$. This may be due to a central void in the disk interior to about 26 AU (Backman \& Paresce 1993). Aumann (1984) suggested that Vega (A0, $7.8 \mathrm{pc}$ ) could have a hot grain component (500 $\mathrm{K})$ with up to $10^{-3}$ of the grain area of the observed component and not violate this limit. The apparent upward trend in the visibility data may be a symptom of resolved flux in the calibrator stars. We have only three object/calibrator pairs for Vega, not enough to test this hypothesis. Our upper limit is a solar-type disk with approximately $3 \times 10^{3}$ times the density of the solar disk. This disk would have $\mathrm{a} \geq 500 \mathrm{~K}$ emitting area of $10^{24} \mathrm{~cm}^{2}$, about $10^{-3}$ of the grain area of the observed component.

61 Cygni $A$ and B.-Though 61 Cygni is close to the Galactic plane and surrounded by cool cirrus emission, Backman, Gillette, \& Low (1986) identified an IRAS point source with this binary system and deduced a far-infrared excess not unlike Vega's. The color temperature of the excess suggests the presence of dust at distances of more than $15 \mathrm{AU}$ from either star. However, these stars are dim (spectral types K5 and K7), and the region of the disk hot enough to emit strongly at $11.6 \mu \mathrm{m}$ is close to the star and difficult to resolve; we could not detect a solar-type dust disk around either of these objects at any density, assuming the COBE/DIRBE model, or unless it had $10^{5}$ times the density of the solar disk, assuming the IRAS model.

70 Oph B.-70 Oph is a binary (types $\mathrm{K} 0$ and $\mathrm{K} 4$ ) with a separation of 24 pixels (2"6). We were able to assemble a power spectrum for B from nine object/calibrator pairs, but the image of A fell on a part of the LWS chip that suffered from many bad pixels and was unusable. The image of A may also have been distorted by off-axis effects. 70 Oph B, like 61 Cygni A and $\mathrm{B}$, is dim, making any dust around it cool and hard to detect at $11.6 \mu \mathrm{m}$.

$\tau$ Ceti.-IRAS could have barely detected a disk with $\sim 1000$ times the emitting area of the solar disk around Tau Ceti (G8, $3.6 \mathrm{pc}$ ), the nearest $\mathrm{G}$ star. We have only three object/calibrator pairs for this object, not enough data to improve on this limit.

We are grateful to Dana Backman, Alycia Weinberger, Keith Matthews, and Eric Gaidos for helpful discussions, and to Keith Matthews and Shri Kulkarni for assistance with the observations. This research has made use of the SIMBAD database, operated at CDS, Strasbourg, France. The observations reported here were obtained at the W. M. Keck Observatory, which is operated by the California Association for Research in Astronomy, a scientific partnership among California Institute of Technology, the University of California, and the National Aeronautics and Space Administration. It was made possible by the generous financial support of the W. M. Keck Foundation.

\section{REFERENCES}

Aumann, H. H. 1984, ApJ, 278, L23

Backman, D. E., Gillette, F. C., \& Low, F. J. 1986, Adv. Space Res., 6,43

Backman, D. E., \& Paresce, F. 1993, in Protostars and Planets III, ed. E. H. Levy \& J. I. Lunine (Tucson: Univ. Arizona Press), 1253

Beichman, C. 1996, JPL Publ. 96-22, A Road Map for the Exploration of Neighboring Planetary Systems (ExNPS) (Pasadena: JPL)
Briggs, R. E. 1962, AJ, 67, 710

Cohen, M., Witteborn, F. C., Carbon, D. F., Davies, J. K., Wooden, D. H., \& Bergman, J. D. 1996, AJ, 112, 2274

Dermott, S. F., Gomes, R. S., Dorda, D. D., Gustafson, B. Å., Jayaraman, S., Xu, Y. L., \& Nicholson, P. D. 1992, in Chaos, Resonance and Collective Dynamical Phenomena in the Solar System, ed. S. Ferraz-Mello (Dordrecht: Kluwer), 333 
Egret, D., Wenger, M., \& Dubois, P. 1991, in Databases and On-line Data in Astronomy, ed. M. A. Albrecht \& D. Egret (Dordrecht: Kluwer), 79

Engelke, C. W. 1990, LWIR Stellar Calibration: Infrared Spectral Curves for 30 Standard Stars. Lincoln Lab. Project Rep. SDP-327

ESA. 1997, The Hipparcos and Tycho Catalogues (ESA SP-1200)

Hanbury Brown, R., Davis, J., \& Allen, L. R. 1974, MNRAS, 167, 121

Jones, B., \& Peutter, R. 1993, Proc. SPIE, 1946, 610
Kelsall, T., et al. 1998, ApJ, in press

Labeyrie, A. 1970, A\&A, 6, 85

Leinert, C., Richter, I., Pitz, E., \& Planck, B. 1981, A\&A, 103, 177

Liou, J. C., \& Zook, H. A. 1996, Icarus, 123, 491

MacQueen, R. M., \& Greeley, B. W. 1995, ApJ, 440, 361

Mann, I., \& MacQueen, R. M. 1993, A\&A, 275, 293

Moshir, M., et al. 1992, Explanatory Supplement to the IRAS Faint Source Survey, Version 2, JPL D-10015 8/92 (Pasadena: JPL)

Robertson, H. P. 1937, MNRAS, 97, 423

Wyatt, S. P., \& Whipple, F. L. 1950, ApJ, 111, 134 